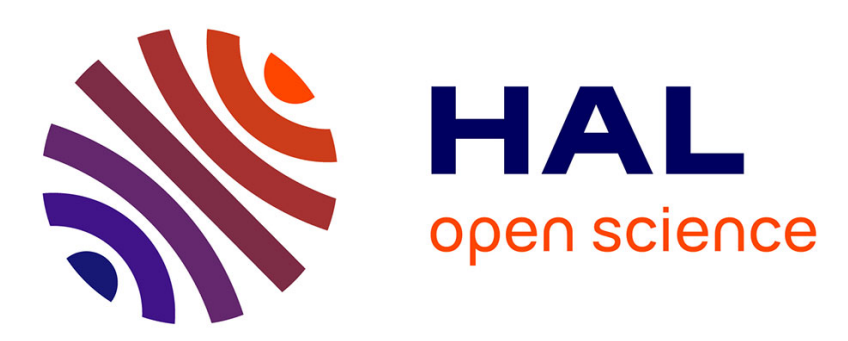

\title{
Shock detection and limiting with discontinuous Galerkin methods for hyperbolic conservation laws
}

Lilia Krivodonova, Jianguo Xin, Jean-François Remacle, Nicolas Chevaugeon, Joseph Flaherty

\section{- To cite this version:}

Lilia Krivodonova, Jianguo Xin, Jean-François Remacle, Nicolas Chevaugeon, Joseph Flaherty. Shock detection and limiting with discontinuous Galerkin methods for hyperbolic conservation laws. Applied Numerical Mathematics, 2004, 48 (3-4), pp.323-338. 10.1016/j.apnum.2003.11.002 . hal-01007288

\section{HAL Id: hal-01007288 \\ https://hal.science/hal-01007288}

Submitted on 25 Jan 2018

HAL is a multi-disciplinary open access archive for the deposit and dissemination of scientific research documents, whether they are published or not. The documents may come from teaching and research institutions in France or abroad, or from public or private research centers.
L'archive ouverte pluridisciplinaire HAL, est destinée au dépôt et à la diffusion de documents scientifiques de niveau recherche, publiés ou non, émanant des établissements d'enseignement et de recherche français ou étrangers, des laboratoires publics ou privés. 


\title{
Shock detection and limiting with discontinuous Galerkin methods for hyperbolic conservation laws
}

\author{
L. Krivodonova ${ }^{\text {a }}$, J. Xin ${ }^{\text {b }}$, J.-F. Remacle ${ }^{c}$, N. Chevaugeon ${ }^{\text {d }}$, J.E. Flaherty ${ }^{\text {d,* }}$ \\ ${ }^{a}$ Courant Institute of Mathematical Sciences, New York University, New York, NY 10012, USA \\ ${ }^{\mathrm{b}}$ Department of Mathematical Sciences, Rensselaer Polytechnic Institute, Troy, NY 12180, USA \\ ${ }^{\mathrm{c}}$ Université Catholique de Louvain-la-Neuve, 1348 Louvain-la-Neuve, Belgium \\ d Scientific Computation Research Center, Rensselaer Polytechnic Institute, Troy, NY 12180, USA
}

We describe a strategy for detecting discontinuities and for limiting spurious oscillations near such discontinuities when solving hyperbolic systems of conservation laws by high-order discontinuous Galerkin methods. The approach is based on a strong superconvergence at the outflow boundary of each element in smooth regions of the flow. By detecting discontinuities in such variables as density or entropy, limiting may be applied only in these regions; thereby, preserving a high order of accuracy in regions where solutions are smooth. Several one- and two-dimensional flow problems illustrate the performance of these approaches.

\section{Introduction}

High-order numerical schemes produce spurious oscillations near discontinuities, which may, indeed, lead to nonlinear (numerical) instabilities and unbounded computational solutions. Less severe oscillations may produce nonphysical solutions, such as negative pressures or temperatures, which would generally lead to physical instabilities. First-order schemes are the only approaches that maintain a monotonic solution structure at discontinuities [10,11]. Unfortunately, these schemes represent the solution with an excessive amount of dissipation. The goal, of course, is to produce a solution with neither excessive diffusion nor spurious oscillations.

Solution or flux limiting strategies [19,20,5,3,17] are designed to restrict or suppress oscillations near discontinuities. Limiting is a nonlinear procedure even for linear problems so the numerical scheme

\footnotetext{
* Corresponding author.

E-mail address: flaherje@ cs.rpi.edu (J.E. Flaherty).
} 
may become both high order and monotonic. Common limiting procedures are based on comparing elemental solution features, such as slopes or curvatures, with those of neighboring elements [5,9]. When slopes, etc. exceed a specified threshold, spurious behavior is deemed present and the range of the excursions is limited. Unfortunately, such limiters frequently identify regions near smooth extrema as requiring limiting [3] and this typically results in a reduction of the optimal high-order convergence rate. It would, thus, be helpful to distinguish regions where solutions are smooth and discontinuous. With such knowledge, limiting would only be used near discontinuities and high-order accuracy would be preserved in smooth regions. The desired procedure will be called a "discontinuity detector".

Recent investigations $[1,8,15]$ have identified a "strong" superconvergence property of the discontinuous Galerkin method (DGM) whereby smooth solutions at outflow boundaries of elements converge at a high $\mathcal{O}\left(h^{2 p+1}\right)$ rate on elements of size $h$ with piecewise-polynomial approximations of degree $p \geqslant 0$. This is to be compared to a global (e.g., $\left.\mathcal{L}^{2}\right)$ convergence rate of $\mathcal{O}\left(h^{p+1}\right)$. With this, we show (Section 3 ) that jumps in the solution across neighboring element edges have different magnitudes in the presence or absence of discontinuities and these may be used to detect solution discontinuities. Dolejsi and Feistauer [7] used a similar strategy to detect shocks between first- and second-order solutions. Likewise, Karni et al. [13] suggested a related indicator based on the weak local truncation error analysis of a central-upwind scheme.

When limiting is needed, we use a slope [5,9] and curvature [2] limiting scheme (Section 3.2) in one and two dimensions, respectively. With limiting restricted to the vicinity of discontinuities, other limiting strategies $[19,20,5,3,17]$ would also suffice. We use the discontinuity detection scheme with both density and entropy as detection variables and show (Section 4) that entropy detection is slightly more robust in that it reliably identifies contact discontinuities as well as shocks for several one- and twodimensional problems. Limiting only where suggested by either detection strategy is far superior than limiting everywhere. Diffusion is limited to discontinuity regions and high-order accuracy is maintained where solutions are smooth.

\section{Formulation}

We consider the solution of hyperbolic conservation laws of the form

$$
\begin{aligned}
& \partial_{t} \mathbf{u}+\operatorname{div} \overrightarrow{\mathbf{F}}(\mathbf{u})=\mathbf{r}(\mathbf{u}), \quad \vec{x} \in \Omega, t>0, \\
& \mathbf{u}=\mathbf{u}^{0}, \quad t=0,
\end{aligned}
$$

with appropriate well-posed boundary data prescribed on $\partial \Omega$. Variables with a superimposed arrow refer to physical vectors in $\Re^{d}, d=1,2,3$, and those in bold type refer to a continuous field in $\left(\mathcal{H}^{1}\right)^{m}$. The flux matrix is

$$
\overrightarrow{\mathbf{F}}(\mathbf{u}):=\left[\vec{F}_{1}(\mathbf{u}), \vec{F}_{2}(\mathbf{u}), \ldots, \vec{F}_{m}(\mathbf{u})\right]
$$

where $\vec{F}_{i}(\mathbf{u})$ is the $i$ th component of the flux $\overrightarrow{\mathbf{F}}$, and

$$
\operatorname{div}:=[\operatorname{div}, \operatorname{div}, \ldots, \operatorname{div}]^{\mathrm{T}},
$$

is the vector valued divergence operator. 
Although the formulation applies to arbitrary conservation laws, our motivation is compressible, inviscid flow governed by the Euler equations. These have the form (1) with

$$
\begin{aligned}
& \mathbf{u}=[\rho, \rho u, \rho v, \rho w, E]^{\mathrm{T}} \\
& \overrightarrow{\mathbf{F}}=\left[\rho \vec{v}, \rho u \vec{v}+P \vec{e}_{x}, \rho v \vec{v}+P \vec{e}_{y}, \rho w \vec{v}+P \vec{e}_{z}, \vec{v}(E+P)\right]
\end{aligned}
$$

where $\rho$ is the fluid density; $\vec{v}$ is the velocity vector with Cartesian components $u, v$, and $w ; E$ is the internal energy; $P$ is the pressure; and $\vec{e}_{x}, \vec{e}_{y}$, and $\vec{e}_{z}$ are unit vectors in the Cartesian coordinate directions. For the computational results of Section 4, we assume the fluid to be an ideal polytropic gas satisfying the equation of state

$$
P=(\gamma-1)\left[E-\frac{\rho\|\vec{v}\|^{2}}{2}\right]
$$

where $\gamma$ is the adiabatic exponent, which was set to 1.4 for the examples.

A DGM formulation [4,5] requires dividing $\Omega$ into a collection of elements

$$
\Omega=\bigcup_{j=1}^{N_{h}} \Omega_{j}
$$

and constructing a Galerkin problem on one element $\Omega_{j}$ by multiplying (1a) by a test function $\mathbf{v} \in$ $\left(\mathcal{L}^{2}\left(\Omega_{j}\right)\right)^{m}$, integrating the result on $\Omega_{j}$, and using the divergence theorem to obtain

$$
\left(\mathbf{v}, \partial_{t} \mathbf{u}\right)_{\Omega_{j}}-(\operatorname{gradv}, \overrightarrow{\mathbf{F}}(\mathbf{u}))_{\Omega_{j}}+\left\langle\mathbf{v}, \overrightarrow{\mathbf{F}}_{n}\right\rangle_{\partial \Omega_{j}}=(\mathbf{v}, \mathbf{r})_{\Omega_{j}}, \quad \forall \mathbf{v} \in\left(\mathcal{L}^{2}\left(\Omega_{j}\right)\right)^{m}
$$

The $\mathcal{L}^{2}$ volume and surface inner products are

$$
(\mathbf{v}, \mathbf{u})_{\Omega_{j}}=\int_{\Omega_{j}} \mathbf{v}^{\mathrm{T}} \mathbf{u} \mathrm{d} \tau, \quad\langle\mathbf{v}, \mathbf{u}\rangle_{\partial \Omega_{j}}=\int_{\partial \Omega_{j}} \mathbf{v}^{\mathrm{T}} \mathbf{u} \mathrm{d} \sigma .
$$

To complete the numerical formulation, $\mathbf{u}$ is approximated by $\mathbf{U} \in \mathcal{P}_{p}\left(\Omega_{j}\right)$, where $\mathcal{P}_{p}$ consists of polynomials of degree $p$ on $\Omega_{j}$. A basis for $\mathcal{P}_{p}\left(\Omega_{j}\right)$ is chosen to be orthogonal in $\mathcal{L}^{2}[16,8,15]$ on $\Omega_{j}$ and this leads to the Dubiner basis commonly used with spectral methods [14]. With a discontinuous basis, the normal flux $\mathbf{F}_{n}=\overrightarrow{\mathbf{F}}(\mathbf{u}) \cdot \vec{n}$, where $\vec{n}$ is the normal vector to $\partial \Omega_{j}$, is not defined on $\partial \Omega_{j}$. The usual strategy is to approximate it by a numerical flux $\mathbf{F}_{n}\left(\mathbf{U}_{j}, \mathbf{U}_{n b_{j}}\right)$ that depends on the solution $\mathbf{U}_{j}$ on $\Omega_{j}$ and $\mathbf{U}_{n b_{j}}$ on the neighboring element $\Omega_{n b_{j}}$ sharing the portion of the boundary $\partial \Omega_{j, n b_{j}}$ common to both elements. Several choices are possible [4,5] and in this investigation we use Roe's flux [17] obtained by solving a linearized Riemann problem across the interface between the two elements. Volume and boundary inner products appearing in (4) are done by Gaussian quadrature of orders $2 p$ and $2 p+1$, respectively. Time integration is performed by an explicit total variation bounded (TVB) Runge-Kutta integration scheme [5]. 


\section{Discontinuity detection and limiting}

\subsection{Discontinuity detection}

For a given linear or nonlinear problem, partition $\partial \Omega_{j}$ into portions $\partial \Omega_{j}^{-}$and $\partial \Omega_{j}^{+}$where, respectively, the flow is into $(\vec{v} \cdot \vec{n}<0)$ and out of $(\vec{v} \cdot \vec{n}>0) \Omega_{j}$. As noted, smooth DGM solutions of (4) exhibit a strong superconvergence phenomena at outflow boundaries $[1,15]$ such that

$$
\frac{1}{\left|\partial \Omega_{j}^{+}\right|} \int_{\partial \Omega_{j}^{+}}\left(Q_{j}-q\right) \mathrm{d} s=\mathcal{O}\left(h^{2 p+1}\right)
$$

where $\left|\partial \Omega_{j}^{+}\right|$is the length (area) of $\partial \Omega_{j}^{+}, q$ is a solution component or a derived quantity from a solution component (e.g., a characteristic variables, the density, or the entropy), and $Q_{j}$ is the discontinuous Galerkin value of $q$ on $\Omega_{j}$.

To use this information as a discontinuity detector, consider the jump in $Q_{j}$ across the inflow edges (faces) of $\Omega_{j}$ and examine

$$
I_{j}=\int_{\partial \Omega_{j}^{-}}\left(Q_{j}-Q_{n b_{j}}\right) \mathrm{d} s=\int_{\partial \Omega_{j}^{-}}\left(Q_{j}-q\right) \mathrm{d} s+\int_{\partial \Omega_{n b_{j}}^{+}}\left(q-Q_{n b_{j}}\right) \mathrm{d} s .
$$

Using (5), the second integral is $\mathcal{O}\left(h^{2(p+1)}\right)$ while the first, across the inflow boundary, is $\mathcal{O}\left(h^{p+2}\right)[1,15]$. Thus, $I_{j}=\mathcal{O}\left(h^{p+2}\right)$ across edges or faces where the solution is smooth. If $q$ is discontinuous in the immediate vicinity of $\partial \Omega_{j}$, then either or both of $q-Q_{j}$ and $q-Q_{n b_{j}}$ are $\mathcal{O}(1)$; hence,

$$
I_{j}= \begin{cases}\mathcal{O}\left(h^{p+2}\right), & \text { if }\left.q\right|_{\partial \Omega_{j}} \text { is smooth, } \\ \mathcal{O}(h), & \text { if }\left.q\right|_{\partial \Omega_{j}} \text { is discontinuous. }\end{cases}
$$

We construct a discontinuity detector by normalizing $I_{j}$ relative to an "average" $\mathcal{O}\left(h^{(p+1) / 2}\right)$ convergence rate and the solution on $\Omega_{j}$ to obtain

$$
\mathcal{I}_{j}=\frac{\left|\int_{\partial \Omega_{j}^{-}}\left(Q_{j}-Q_{n b_{j}}\right) \mathrm{d} s\right|}{h^{(p+1) / 2}\left|\partial \Omega_{j}^{-}\right|\left\|Q_{j}\right\|} .
$$

In examples, we choose $h$ as the radius of the circumscribed circle in element $\Omega_{j}$, and use a maximum norm based on local solution maxima at integration points in two dimensions and an element average in one dimension.

Using (7), $\mathcal{I}_{j} \rightarrow 0$ as either $h \rightarrow 0$ or $p \rightarrow \infty$ in smooth solution regions, whereas $\mathcal{I}_{j} \rightarrow \infty$ near a discontinuity. Thus, the discontinuity detection scheme is

$$
\left\{\begin{array}{l}
\text { if } \mathcal{I}_{j}>1, \quad q \text { is discontinuous, } \\
\text { if } \mathcal{I}_{j}<1, \quad q \text { is smooth. }
\end{array}\right.
$$

\subsection{Limiting}

With limiting only used near discontinuities, we need not be as concerned with maintaining a high order of accuracy; thus, we focus on the slope limiting procedure introduced by Cockburn and Shu [5] 
for one-dimensional problems and the curvature limiting schemes of Barth and Jespersen [2] in multiple dimensions. Slope limiting compares solution gradients on $\Omega_{j}$ with average solution gradients on neighboring elements. The computed and average gradients are compared and elemental slopes are restricted to the range spanned by neighboring averages when all have the same sign. Slopes are set to zero should signs disagree $[5,19,20]$. For scalar problems on a uniform mesh, these conditions may be summarized by letting

$$
S_{\xi j}=U_{j}(\xi, t)-\bar{U}_{j}, \quad \xi=-1,1,
$$

where $\bar{U}_{j}$ is the solution average on $\Omega_{j}$. We calculate modified slopes at the ends $x_{j-1}$ and $x_{j}$ of $\Omega_{j}$ as

$$
\begin{aligned}
& S_{1, j}=\operatorname{minmod}\left(U_{j}\left(x_{j}, t\right)-\bar{U}_{j}, \Delta \bar{U}_{j}, \nabla \bar{U}_{j}\right), \\
& S_{-1, j}=\operatorname{minmod}\left(\bar{U}_{j}-U_{j}\left(x_{j-1}, t\right), \Delta \bar{U}_{j}, \nabla \bar{U}_{j}\right),
\end{aligned}
$$

where

$$
\Delta \bar{U}_{j}:=\bar{U}_{j+1}-\bar{U}_{j}, \quad \nabla \bar{U}_{j}:=\bar{U}_{j}-\bar{U}_{j-1},
$$

and

$$
\operatorname{minmod}(a, b, c):= \begin{cases}\operatorname{sgn}(a) \min (|a|,|b|,|c|), & \text { if } \operatorname{sgn}(a)=\operatorname{sgn}(b)=\operatorname{sgn}(c), \\ 0, & \text { otherwise. }\end{cases}
$$

The modified slopes are used to alter solution coefficients (cf., e.g., (12)). Assuming the average solution $\bar{U}_{j}$ remains unchanged, (11) provide two equations for $p$ unknowns; thus, unique solutions do not exist when $p>2$. The problem can be closed by either setting higher order coefficients to zero or using $\mathcal{L}^{2}$ projection [5].

The resulting solution is total variational diminishing (TVD), which implies that the order of accuracy is reduced near smooth extrema [5]. As noted, avoiding limiting in smooth solution regions avoids this loss. Another possibility is the limiting of the highest order coefficients through the computation of moments [3].

With vector systems, the simple strategy of applying limiting component-wise is not TVD [4]. Instead, limiting should be applied to characteristic variables obtained by diagonalizing the system Jacobian $[3,4]$.

Turning now to solution limiting on unstructured two-dimensional triangular meshes, consider a (scalar) solution represented on element $\Omega_{j}$ in terms of an orthogonal basis $\left\{\varphi_{i}(\vec{x})\right\}_{k=1}^{N_{p}}[8]$ as

$$
U_{j}(\vec{x}, t)=\sum_{k=1}^{N_{p}} c_{k, j}(t) \varphi_{k}(\vec{x})
$$

Following Barth and Jespersen [2], slopes are limited so that the solution at the integration points $\vec{x}_{i}^{*}$, $i=1,2, \ldots, K_{\partial \Omega_{j}}$ on $\partial \Omega_{j}$ is in the range spanned by the neighboring solution averages. The modified solution for piecewise-linear polynomials $(p=1)$ is

$$
U_{j}(\vec{x}, t)=c_{1, j}(t) \varphi_{1}(\vec{x})+\alpha\left[c_{2, j}(t) \varphi_{2}(\vec{x})+c_{3, j}(t) \varphi_{3}(\vec{x})\right],
$$

where

$$
\alpha=\min _{1 \leqslant i \leqslant K_{\partial \Omega_{j}}} \max \left(\alpha_{i}, 0\right),
$$




$$
\alpha_{i}= \begin{cases}\frac{M-\bar{U}_{j}}{U_{j}\left(\vec{x}_{i}^{*}\right)-\bar{U}_{j}}, & \text { if } U_{j}\left(\vec{x}_{i}^{*}\right)-M>0, \\ \frac{m-\bar{U}_{j}}{U_{j}\left(\vec{x}_{i}^{*}\right)-\bar{U}_{j}}, & \text { if } U_{j}\left(\vec{x}_{i}^{*}\right)-m<0, \\ 1, & \text { otherwise, }\end{cases}
$$

and $m$ and $M$ are the minimum and maximum solution averages on the elements sharing edges with $\Omega_{j}$. For $p>1$, we set the higher-order coefficients in (12) to zero and apply (13) to the remaining solution whenever $\alpha \neq 1$. Applying limiting to characteristic variables may produce negative pressures; thus, rendering the solution unstable. Should such nonphysical solutions result, we set $U_{j}=\bar{U}_{j}$.

\section{Computational results}

We apply the discontinuity detection and limiting procedures (Section 3) to several one- and twodimensional examples involving the Euler equations (2).

\subsection{One-dimensional examples}

Example 1. Consider a shock tube of unit length with a membrane at $x=0.5$ separating gas with the pressures and densities

$$
(\rho, u, P)(x, 0)= \begin{cases}(1,0,1), & 0 \leqslant x<0.5, \\ (0.1,0,0.01), & 0.5 \leqslant x \leqslant 1 .\end{cases}
$$

The membrane is ruptured at time $t=0$ and the solution breaks into a shock wave moving into the lowpressure gas, a rarefaction wave expanding into the high-pressure gas, and a contact surface separating the two [21].

We solved problems using density and entropy as discontinuity detection variables. Densities are shown in Fig. 1 at $t=0.25$ for computations performed with piecewise-quadratic ( $p=2$ ) polynomials on meshes having 50 uniform elements. Those elements identified for limiting (Section 3.2) are indicated with a plus sign at unity. Numerical solutions are shown with three diamonds per element. We additionally show the values of the two discontinuity detectors on the lower portion of Fig. 1.

The solutions obtained using the two discontinuity detection schemes are nearly identical (Fig. 1, top). Both discontinuity detection variables correctly identified the shock region. The indicators are well above unity near the shock and approximately zero in smooth flow regions (Fig. 1, bottom). The density detection strategy applied no limiting in the contact region. This was not always the case and the density detector exceeded its threshold at some times. At any rate, limiting appears not to be necessary in the contact region at this time.

Additional solutions were computed using the two discontinuity detection strategies and the more traditional strategy of limiting everywhere. Computations were performed with $p=2$ on uniform meshes with 100, 200, and 500 elements and the $\mathcal{L}^{2}$ errors in density are shown in Table 1 at $t=0.25$. The results differ by less than $5 \%$ on the coarsest and $3 \%$ on the finest meshes. This solution does not possess smooth extrema; thus, discontinuity detection does not provide significant accuracy advantages. However, the simple structure of the solution and known analytical solution provide a valuable frame of reference. 

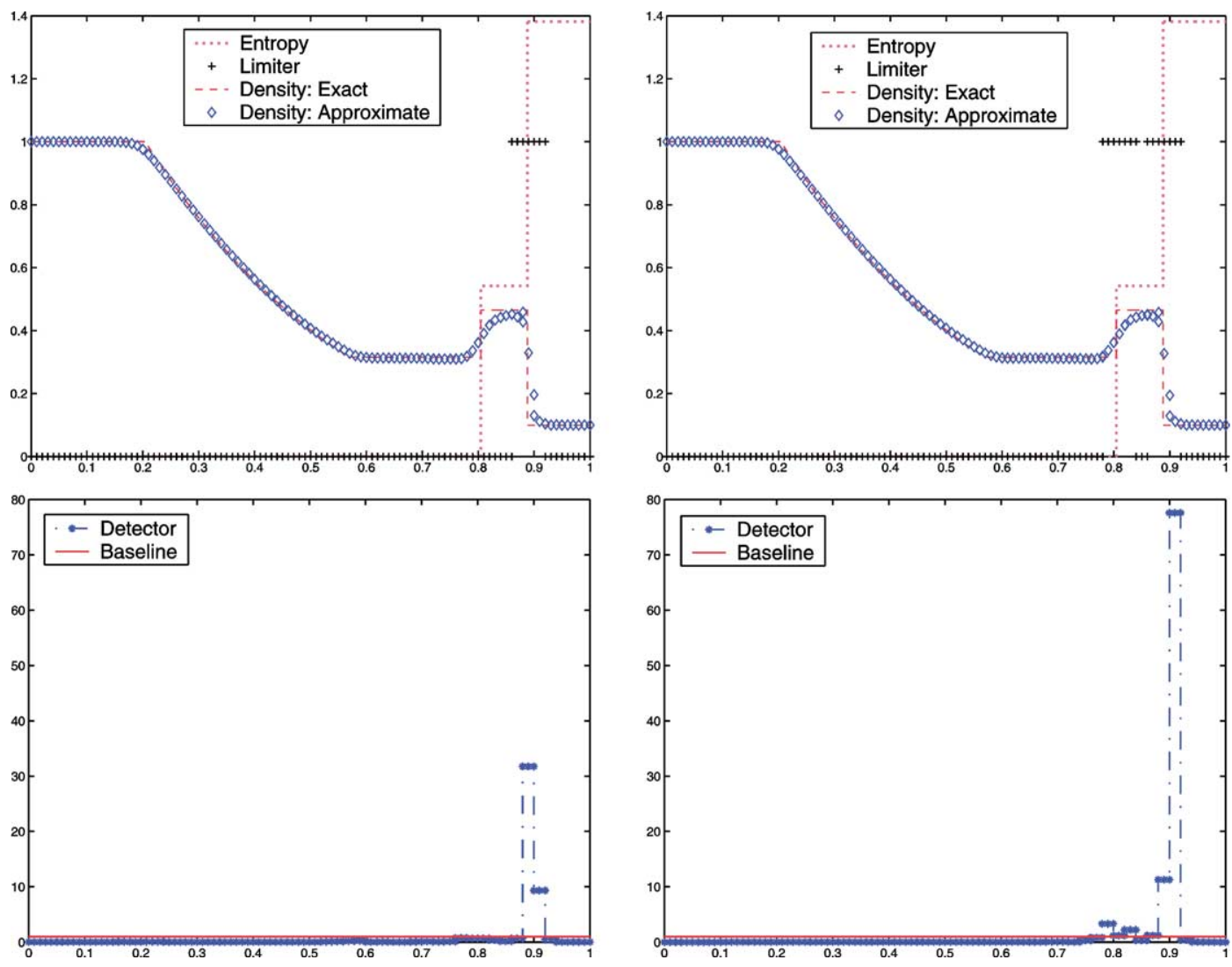

Fig. 1. Density for Example 1 at $t=0.25$ computed with $p=2$ and $N_{h}=50$ using density detection (upper left) and entropy detection (upper right). Elements where limiting occurred are marked with + sign at unity. Values of the density (lower left) and entropy (lower right) detectors.

Table 1

$\mathcal{L}^{2}$ error in density for Example 1 at $t=0.25$ for computations performed with $p=2$ and $N_{h}=50,100,200$, and 500 using moment limiting, density detection, and entropy detection

\begin{tabular}{rccc}
\hline$N_{h}$ & \multicolumn{3}{c}{ Density error } \\
\cline { 2 - 4 } & Limiting & Density detection & Entropy detection \\
\hline 50 & $1.76 \mathrm{e}-3$ & $1.70 \mathrm{e}-3$ & $1.69 \mathrm{e}-3$ \\
100 & $9.40 \mathrm{e}-4$ & $9.28 \mathrm{e}-4$ & $9.17 \mathrm{e}-4$ \\
200 & $4.76 \mathrm{e}-4$ & $4.73 \mathrm{e}-4$ & $4.64 \mathrm{e}-4$ \\
500 & $1.82 \mathrm{e}-4$ & $1.81 \mathrm{e}-4$ & $1.78 \mathrm{e}-4$ \\
\hline
\end{tabular}

Example 2. Consider the Euler equations (2) subject to the initial data [18]

$$
(\rho, u, P)(x, 0)= \begin{cases}(3.857143,-0.920279,10.33333), & x \leqslant 0, \\ (1+0.2 \sin (5 x),-3.549648,1.00000), & 0<x<10, \\ (1.0000,-3.549648,1.00000), & x \geqslant 10 .\end{cases}
$$

This example involves the interaction of a stationary shock at $x=0$ with a leftward-moving flow having a sinusoidal density variation. As the density perturbation passes through the shock, it produces oscillations 

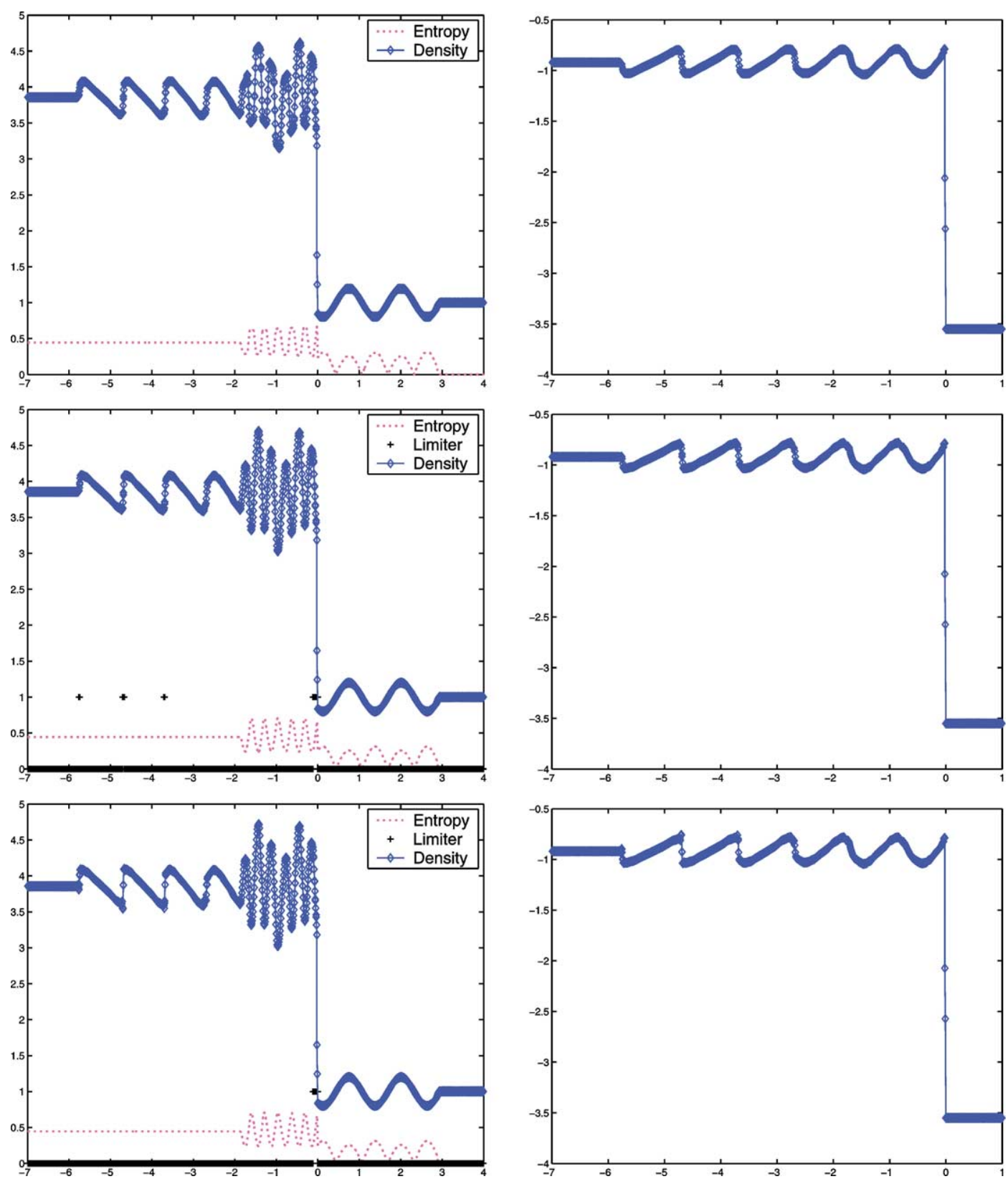

Fig. 2. Entropy-density (left) and velocity (right) for Example 2 at $t=2.0$ for computations performed with $p=2$ and $N_{h}=1024$ using moment limiting (top), density detection (middle), and entropy detection (bottom). Elements where limiting occurred are marked with + sign at unity.

developing into shocks of smaller amplitude. A poor limiting strategy would damp the oscillations and, thus, the aim is to avoid this.

We solved $(2,15)$ on $-10 \leqslant x \leqslant 10,0<t \leqslant 2$ using a 1024-element mesh with $p=2$ using limiting everywhere, limiting where indicated by density detection, and limiting where indicated by entropy 


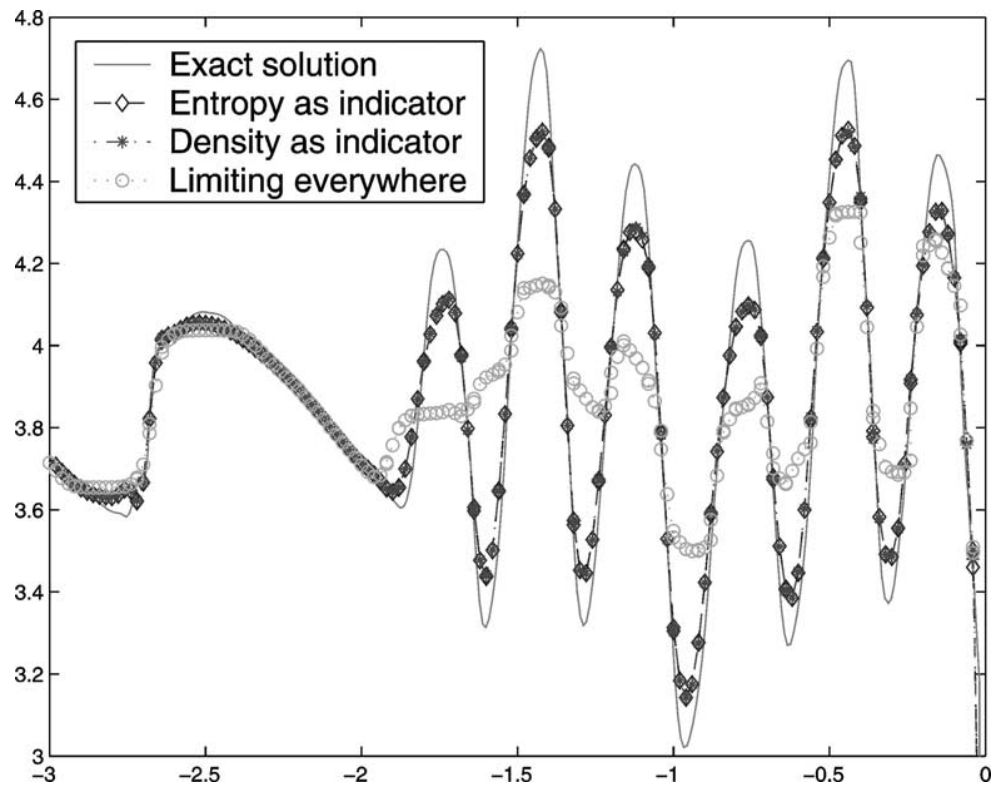

Fig. 3. Density for Example 2 at $t=2.0$ on $-3 \leqslant x \leqslant 0$ computed with $p=2$ and $N_{h}=500$ using limiting everywhere, density detection, and entropy detection.

detection and present results in Fig. 2. Density detection identified both the strong shock at $x=0$ and the weaker ones on the negative axis. The shocks are well-captured without visible overshoots or undershoots. Entropy detection identified the strong shock but did not activate near the weaker shocks because the jumps at these locations were too small. This has resulted in slight loss of monotonicity near these weaker shocks. Universal application of limiting has dissipated the solution in the oscillatory region relative to those with density and entropy detection.

To amplify differences, we compare solutions with universal limiting and with the two discontinuity detection strategies on a coarser 500-element mesh with $p=2$ and present results for the density at $t=2$ in the high-frequency region $(-3 \leqslant x \leqslant 0)$ in Fig. 3 . The computed solution with $N_{h}=1024, p=2$, and entropy detection is regarded as an exact solution.

The solution with limiting everywhere has too much dissipation and has greatly reduced the amplitude of the oscillations. Both density and entropy detection gave similar results with sharp detection of the strong shock and its confinement to two elements. Neither detection scheme located the weaker shocks and, thus, they produced some minor overshoots.

Example 3. Consider the Euler equations (2) with the initial data

$$
(\rho, u, P)(x, 0)= \begin{cases}(1,0,1000), & 0 \leqslant x<0.1, \\ (1,0,0.01), & 0.1 \leqslant x<0.9, \\ (1,0,100), & 0.9 \leqslant x \leqslant 1,\end{cases}
$$

where the solution involves interactions between strong shocks, expansions, and contact discontinuities [22].

An exact solution of this problem is not known, so, following Woodward and Colella [22], comparisons were done relative to a numerical solution computed with $N_{h}=3072$ uniform elements, $p=2$, and entropy discontinuity detection. We compare solutions for the density with those obtained 

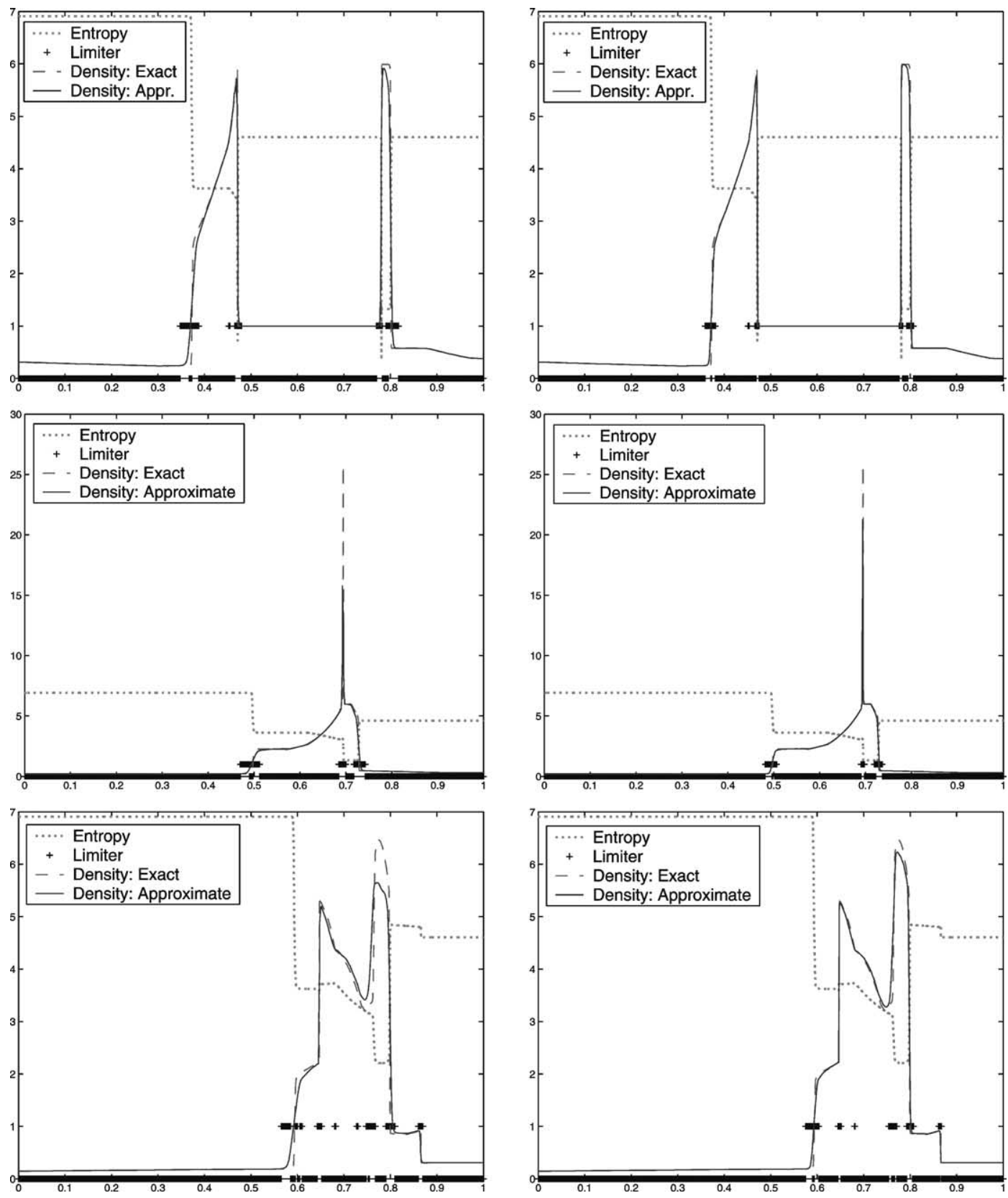

Fig. 4. Entropy-density for Example 3 at $t=0.016$ (top), 0.028 (middle), and 0.038 (bottom) for computations performed with $p=2, N_{h}=400$ (left), and $N_{h}=800$ (right) using entropy detection. Elements where limiting occurred are marked with + sign at unity.

using $N_{h}=400$ and 800, $p=2$, and entropy detection at $t=0.016,0.028$, and 0.038 in Fig. 4. Solutions calculated with density discontinuity detection and limiting everywhere followed the trends of the two previous examples and have not been shown. 
Discontinuities are identified and limiting is confined to these regions. Computational values of the density with $N_{h}=400$ and 800 are showing some dissipative effects near extrema due to diffusion of the contact surfaces. The resolution of these (third-order) solutions is significantly higher than that obtained with a (third-order) WENO-RF-3 scheme and is close to that obtained by a (fourth-order) WENO-RF-4 scheme on the same meshes [12].

\subsection{Two-dimensional examples}

Example 4. The propagation of sound in air satisfies the linearization of the Euler equations (2)

$$
\frac{\partial}{\partial t}\left[\begin{array}{l}
P \\
u \\
v
\end{array}\right]+\frac{\partial}{\partial x}\left[\begin{array}{c}
\rho_{0} c^{2} u \\
P / \rho_{0} \\
0
\end{array}\right]+\frac{\partial}{\partial y}\left[\begin{array}{c}
\rho_{0} c^{2} v \\
0 \\
P / \rho_{0}
\end{array}\right]=\mathbf{0},
$$

where $\rho_{0}$ is the density of the fluid, $c$ is the speed of sound in the fluid, and $P, u$, and $v$ are now regarded as perturbations to the pressure and velocity components, respectively.

We solve (17) using the DGM with a numerical flux that is the exact solution of an associated onedimensional Riemann problem. Thus, consider an interface with normal $\vec{n}=\left(n_{x}, n_{y}\right)$ separating elements having pressures and normal velocities $P^{l}, v_{n}^{l}$ and $P^{r}, v_{n}^{r}$. The solution of the associated Riemann problem is

$$
P=\frac{P^{l}+P^{r}}{2}+\rho_{0} c \frac{v_{n}^{l}-v_{n}^{r}}{2}, \quad v_{n}=\frac{v_{n}^{l}+v_{n}^{r}}{2}+\frac{1}{\rho_{0} c} \frac{P^{l}-P^{r}}{2},
$$

and the numerical flux is

$$
\mathbf{F}_{n}=\frac{1}{2}\left[\begin{array}{c}
\rho_{0} c^{2}\left(v_{n}^{l}+v_{n}^{r}\right) \\
\left(P^{l}+P^{r}\right) n_{x} / \rho_{0} \\
\left(P^{l}+P^{r}\right) n_{y} / \rho_{0}
\end{array}\right]+\frac{1}{2} c\left[\begin{array}{c}
\left(P^{l}-P^{r}\right) \\
\left(v_{n}^{l}-v_{n}^{r}\right) n_{x} \\
\left(v_{n}^{l}-v_{n}^{r}\right) n_{y}
\end{array}\right] .
$$

We solve an acoustic problem in a $0.1 \times 1$ rectangular cavity. The left edge $(x=0)$ of the domain is a vibrating wall prescribing the velocity

$$
u(0, t)=1+\frac{1}{2} \sin (2 \pi f t),
$$

with the frequency $f=1500 \mathrm{~Hz}$. An admittance boundary condition

$$
u(1, t)=\frac{P(1, t)}{\rho_{0} c},
$$

is used at the right edge $(x=1)$ of the domain. Horizontal edges $y= \pm 0.05$ are rigid walls; hence,

$$
v( \pm 0.05, t)=0 \text {. }
$$

The medium has the sound speed $c=340 \mathrm{~m} / \mathrm{sec}$ and density $\rho_{0}=1.224 \mathrm{~kg} / \mathrm{m}^{3}$. The wavelength is $\lambda=c / f=0.2266 \mathrm{~m}$. The gas inside the cavity is initially at rest, so $P=u=v=0$. The solution of the problem features smooth oscillations and a discontinuity at $x_{d}=c t$.

The solution is independent of $y$ and, hence, one dimensional; however, we solve it on an unstructured triangular mesh (Fig. 5) using elements with edges of length 0.02 . This gives a mesh with approximatively 10 elements per wavelength, which is the minimum resolution typically required for a second-order $(p=1)$ method. 


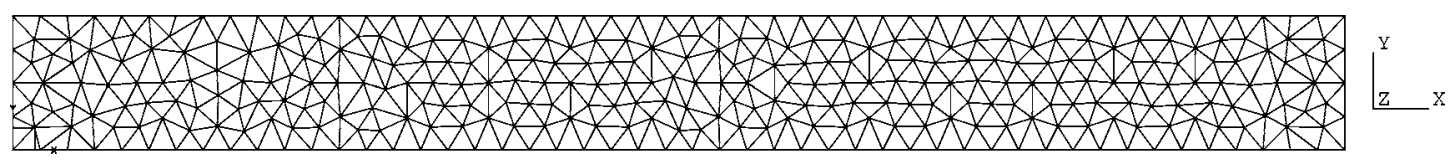

Fig. 5. Mesh used for the acoustic cavity of Example 4.

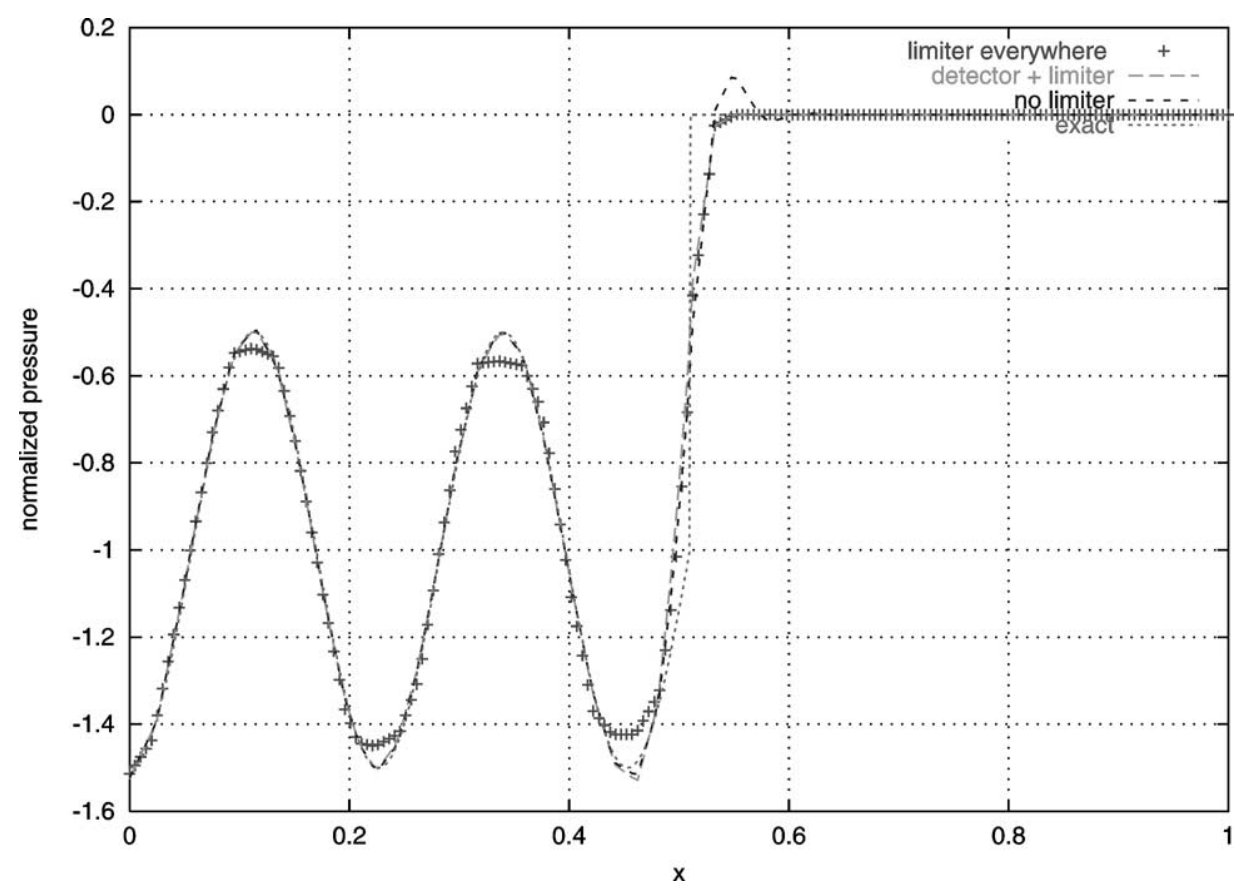

Fig. 6. Normalized pressure $P / \rho_{0} c$ vs. $x$ at $t=1.5 \times 10^{-3}$ for Example 4 with $f=1500 \mathrm{~Hz}$ and $p=1$. The exact solution, solution with limiting everywhere, solution with no limiter, and solution with the detector-limiting strategy are shown.

Results in Fig. 6 show the normalized pressure $P / \rho_{0} c$ as a function of $x$ at time $t=1.5 \times 10^{-3}$. With limiting applied everywhere, smooth extrema are flattened and the amplitude of the wave is decreased. Convergence occurs at a reduced first-order rate. Without limiting, oscillations develop near $x=x_{d}$ but the solution is accurate at smooth extrema. The solution with discontinuity detection only applied limiting near the discontinuity; thus, producing an optimal result without spurious oscillations and excessive diffusion near local extrema.

Next consider the solution of a problem with $f=5000 \mathrm{~Hz}(\lambda=0.068)$ using piecewise-cubic polynomials $(p=3)$ on the mesh of Fig. 5. Thus, there are approximately three elements per wavelength; however, cubic polynomials can capture sinusoidal data with three elements per wavelength.

Results shown in Fig. 7 indicate that limiting everywhere has failed to resolve the high-frequency portion of the solution and renders the use of a high-order approximation useless. Accuracy has been reduced to first order. The detector-limiter procedure does a much better job with only slight dissipation noticed in the oscillatory region. Near the discontinuity, the solution with no limiting can re-establish itself to the continuous solution with less spread than the limited solutions. It does, however, produce spurious oscillations. Clearly, we would like to decrease the spatial zone where limiting is applied. 


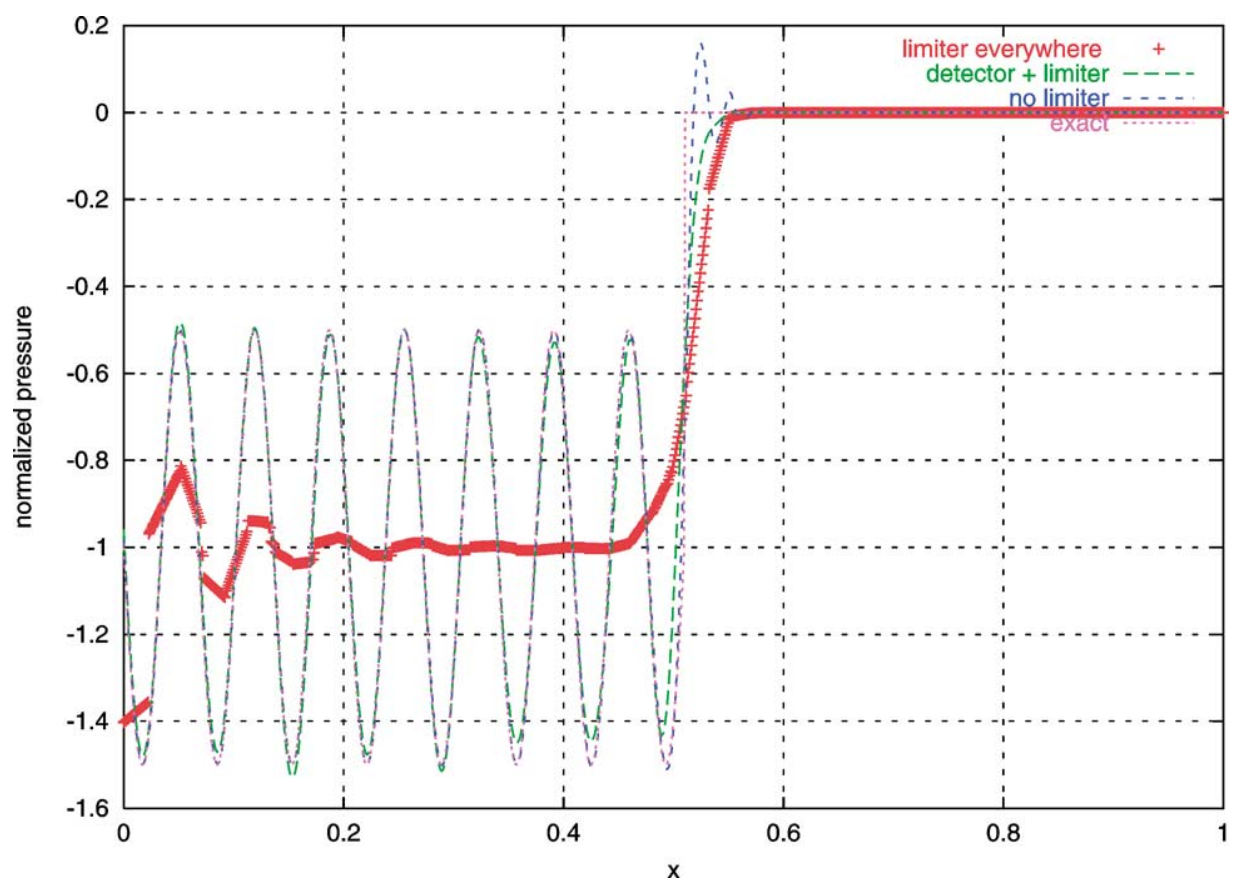

Fig. 7. Normalized pressure $P / \rho_{0} c$ vs. $x$ at $t=1.5 \times 10^{-3}$ for Example 4 with $f=5000 \mathrm{~Hz}$ and $p=3$. The exact solution, solution with limiting everywhere, solution with no limiter, and solution with the detector-limiting strategy are shown.

At present, this can be done using adaptive $h$-refinement, where the mesh is refined in the vicinity of discontinuities.

Example 5. We consider the reflection of a Mach 10 planar shock by a wedge having a half-angle of $30^{\circ}$. This problem, satisfying the two-dimensional Euler equations (2), is referred to as a double Mach reflection and provides a popular benchmark [22,6,12]. To avoid geometrical complications, the $4 \times 1$ rectangular computational domain is rotated to lie along the upper surface of the wedge. With this, the Mach 10 shock is initially placed at $x=1 / 6, y=0$ at $60^{\circ}$ relative to the $x$-axis of the computational domain. The surface of the wedge lies at the bottom of the computational domain for $x \geqslant 1 / 6, y=0$ and is assumed to be a reflecting boundary. Boundary conditions at the top $(y=1)$ correspond to the exact motion of a Mach 10 shock. Physical parameters for the gas ahead of the shock are $P_{1}=1$ and $\rho_{1}=1.4$. The Rankine-Hugoniot relations

$$
\begin{aligned}
& v_{s}=M_{s} \sqrt{\gamma P_{1} / \rho_{1}}=10, \quad P_{2} / P_{1}=\left(2 \gamma M_{s}^{2}-(\gamma-1)\right) /(\gamma+1), \\
& \rho_{2} / \rho_{1}=(\gamma+1) M_{s}^{2} /\left((\gamma-1) M_{s}^{2}+2\right), \quad \rho_{1} v_{s}=\rho_{2}\left(v_{s}-v_{2}\right),
\end{aligned}
$$

define the post shock conditions.

Problems are solved on uniform meshes with spacings $\Delta x=\Delta y=1 / 30$ and $\Delta x=\Delta y=1 / 60$ using limiting everywhere and limiting with entropy discontinuity detection. Density and pressure contours are shown in Figs. 8 and 9 at $t=0.2$ for computations performed with $p=1$ on the two meshes. Contours are plotted using the discontinuous solutions with no smoothing. 

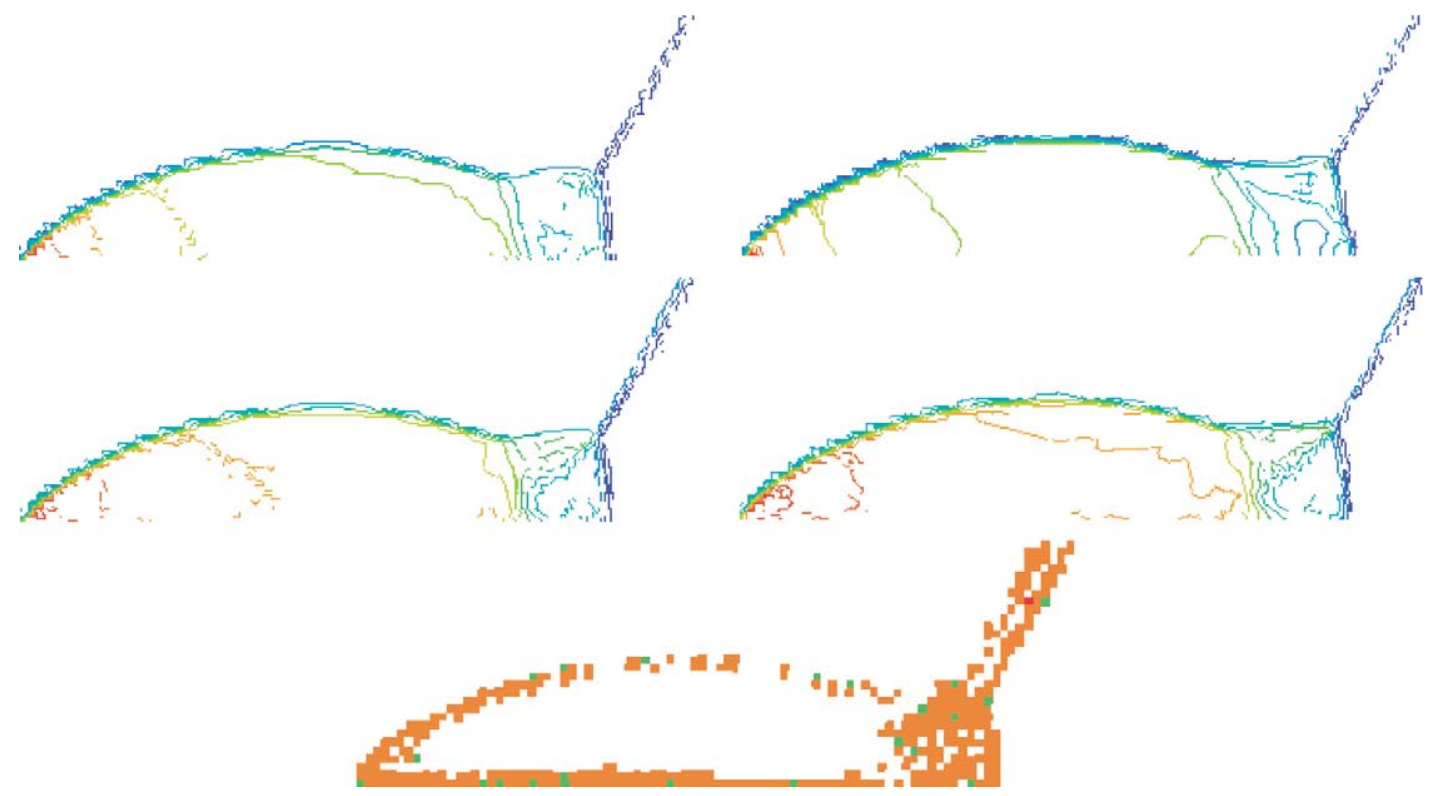

Fig. 8. Pressure (top) and density (middle) contours obtained with limiting everywhere (left) and where indicated by entropy detection (right) for Example 5 at $t=0.2$ with $\Delta x=\Delta y=1 / 30$ and $p=1$. Elements where limiting was applied with entropy detection are marked on the figure at the bottom.

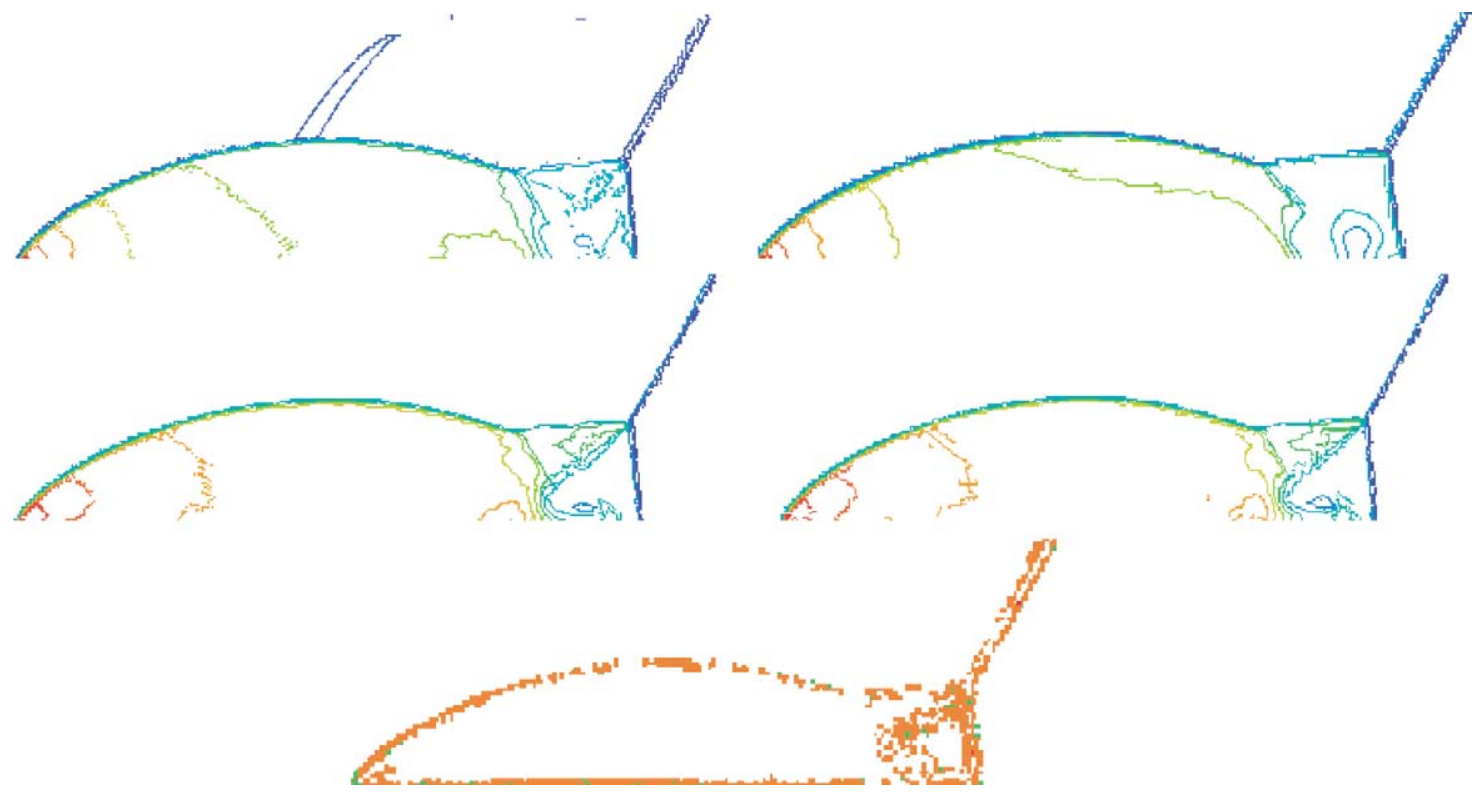

Fig. 9. Pressure (top) and density (middle) contours obtained with limiting everywhere (left) and where indicated by entropy discontinuity detection (right) for Example 5 at $t=0.2$ with $\Delta x=\Delta y=1 / 60$ and $p=1$. Elements where limiting was applied with entropy detection are marked on the figure at the bottom. 
With the entropy detection strategy, the contact surface that emanates from the triple shock intersection and forms the jet in the density profile is well resolved on both the coarser and finer meshes. The weak shock from the main reflected shock to the triple point is also sharp on both meshes, but is better resolved with entropy detection than with limiting everywhere. The jet formed by the double Mach reflection is clear on the finer mesh and less so on the coarser one. The pressure contours in region containing the jet are well resolved on both the coarse and fine mesh. The pressure contours on the finer mesh exhibit a nonphysical structure near the main reflecting shock when limiting everywhere. This is not present when using entropy detection. The contact region is clear and sharp on the refined mesh.

\section{Discussion and conclusion}

We have developed a strategy for detecting discontinuities when using the DGM to solve hyperbolic systems of conservation laws. The strategy is based on a posteriori error estimates $[1,15]$ involving jumps in solution quantities across element boundaries. With successful detection of discontinuities, solution or flux limiting, needed to suppress spurious oscillations, may be restricted to those regions containing discontinuities. This strategy is superior to the practice of limiting everywhere, which typically introduces far more diffusion than necessary in smooth flow regions. For flow problems, we have based discontinuity detection on either jumps in the fluid density or entropy. Both work well for a number of standard test problems for the Euler equations. Using entropy as a discontinuity detector works reliably near shocks and contact discontinuities and, generally, performed slightly better than detection with density.

These discontinuity detection strategies have greatly reduced the need for limiting with high-order DGMs. This, in turn, has dramatically improved the performance of the DGM in smooth flow regions where the solution has complex structure. Accuracy near smooth extrema, in particular, is remarkably better without limiting and is maintaining the optimal convergence rate.

There is some arbitrariness associated with threshold value of the discontinuity detector (8). This might be eliminated by scaling relative to approximations obtained for jumps associated with weak shocks [21] or other discontinuities. We will investigate this possibility.

\section{References}

[1] S. Adjerid, K. Devine, J. Flaherty, L. Krivodonova, A posteriori error estimation for discontinuous Galerkin solutions of hyperbolic problems, Comput. Methods Appl. Mech. Engrg. 191 (2002) 1097-1112.

[2] T. Barth, D. Jespersen, The design and application of upwind schemes on unstructured meshes, in: 27th Aerospace Sciences Meeting, Reno, Nevada, AIAA 89-0366, 1989.

[3] R. Biswas, K. Devine, J. Flaherty, Parallel adaptive finite element methods for conservation laws, Appl. Numer. Math. 14 (1994) 255-284.

[4] B. Cockburn, S. Lin, C.-W. Shu, TVB Runge-Kutta local projection discontinuous Galerkin methods for scalar conservation laws III: One dimensional systems, J. Comput. Phys. 84 (1989) 90-113.

[5] B. Cockburn, C.-W. Shu, TVB Runge-Kutta local projection discontinuous Galerkin methods for scalar conservation laws II: General framework, Math. Comp. 52 (1989) 411-435.

[6] P. Colella, H.M. Glaz, Efficient solution algorithms for the Riemann problem for real gases, J. Comput. Phys. 59 (1985) 264-289.

[7] V. Dolejsi, M. Feistauer, On the discontinuous Galerkin method for the numerical solution of compressible high-speed flow, Technical Report MATH-knm-2002/1, Charles University, Prague, 2002. 
[8] J. Flaherty, L. Krivodonova, J.-F. Remacle, M. Shephard, Aspects of discontinuous Galerkin methods for hyperbolic conservation laws, Finite Elements Anal. Design 38 (2002) 889-908.

[9] J. Goodman, R. LeVeque, A geometric approach to high resolution TVD schemes, SIAM J. Numer. Anal. 25 (1988) 268-284.

[10] A. Harten, High resolution schemes for hyperbolic conservation laws, J. Comput. Phys. 49 (1983) $357-393$.

[11] A. Harten, J. Hyman, P. Lax, On finite-difference approximations and entropy conditions for shocks, Comm. Pure Appl. Math. 29 (1976) 297-322.

[12] G.-S. Jiang, C.-W. Shu, Efficient implementation of weighted ENO schemes, J. Comput. Phys. 126 (1996) $202-228$.

[13] S. Karni, A. Kurganov, G. Petrova, A smoothness indicator for adaptive algorithms for hyperbolic systems, J. Comput. Phys. 178 (2002) 323-341.

[14] G. Karniadakis, S. Sherwin, Spectral/hp Element Methods for CFD, Oxford University Press, New York, 1999.

[15] L. Krivodonova, J. Flaherty, Error estimation for discontinuous Galerkin solutions of multidimensional hyperbolic problems, Adv. Comput. Math. 19 (2003) 57-71.

[16] J.-F. Remacle, J. Flaherty, M.S. Shephard, An adaptive discontinuous Galerkin technique with an orthogonal basis applied to compressible flow problems, SIAM Rev. 45 (2003) 53-72.

[17] P. Roe, Approximate Riemann solvers, parameter vectors and difference schemes, J. Comput. Phys. 43 (1981) $357-372$.

[18] C.-W. Shu, S. Osher, Efficient implementation of essentially non-oscillatory shock-capturing schemes, II, J. Comput. Phys. 83 (1989) 32-78.

[19] B. van Leer, Towards the ultimate conservation difference scheme, II, J. Comput. Phys. 14 (1974) $361-367$.

[20] B. van Leer, Towards the ultimate conservation difference scheme, V, J. Comput. Phys. 32 (1979) 1-136.

[21] G. Whitham, Linear and Nonlinear Waves, Wiley, New York, 1974.

[22] P. Woodward, P. Colella, The numerical simulation of two-dimensional fluid flow with strong shocks, J. Comput. Phys. 54 (1984) 115-173. 